\title{
Neutrophil-to-lymphocyte ratio as a prognostic factor for patients with metastatic or recurrent breast cancer treated using capecitabine: a retrospective study
}

Shigemasa Takamizawa, Tatsunori Shimoi", Natsuko Satomi-Tsushita, Shu Yazaki, Toshihiro Okuya, Yuki Kojima, Hitomi Sumiyoshi-Okuma, Tadaaki Nishikawa, Maki Tanioka, Kazuki Sudo, Emi Noguchi and Kan Yonemori

\begin{abstract}
Background: Eribulin or capecitabine monotherapy is the next cytotoxic chemotherapy option for patients with metastatic or recurrent breast cancer who have previously received an anthracycline or a taxane. However, it is unclear what factors can guide the selection of eribulin or capecitabine in this setting, and prognostic factors are needed to guide appropriate treatment selection. The neutrophil-to-lymphocyte ratio (NLR) is a prognostic factor for eribulintreated patients, although it is unclear whether it is a prognostic factor for capecitabine-treated patients. Therefore, we analysed the ability of the NLR to predict oncological outcomes among patients who received capecitabine after previous anthracycline or taxane treatment for breast cancer.
\end{abstract}

Methods: We retrospectively reviewed the medical records of patients with metastatic or recurrent breast cancer who had previously received anthracycline or taxane treatment at the National Cancer Center Hospital between 2007 and 2015. Patients were included if they received eribulin or capecitabine monotherapy as first-line, second-line, or third-line chemotherapy. Analyses of overall survival (OS) and progression-free survival (PFS) were performed according to various factors.

Results: Between 2007 and 2015, we identified 125 eligible patients, including 46 patients who received only eribulin, 34 patients who received only capecitabine, and 45 patients who received eribulin and capecitabine. The median follow-up period was 19.1 months. Among eribulin-treated patients, an NLR of $<3$ independently predicted better OS. Among capecitabine-treated patients, an NLR of $<3$ independently predicted better PFS but not better OS. In addition, a lymphocyte-to-monocyte ratio of $\geq 5$ was associated with better PFS and OS.

Conclusions: To the best of our knowledge, this is the first study to evaluate whether the NLR is a prognostic factor for capecitabine-treated patients with metastatic or recurrent breast cancer. However, the NLR only independently predicted PFS in this setting, despite it being a useful prognostic factor for other chemotherapies.

*Correspondence: tshimoi@ncc.go.jp

Department of Medical Oncology, National Cancer Center Hospital, 5-1-1

Tsukiji, Chuo-Ku, 104-0045 Tokyo, Japan original author(s) and the source, provide a link to the Creative Commons licence, and indicate if changes were made. The images or other third party material in this article are included in the article's Creative Commons licence, unless indicated otherwise in a credit line to the material. If material is not included in the article's Creative Commons licence and your intended use is not permitted by statutory regulation or exceeds the permitted use, you will need to obtain permission directly from the copyright holder. To view a copy of this licence, visit http://creativecommons.org/licenses/by/4.0/. The Creative Commons Public Domain Dedication waiver (http://creativeco mmons.org/publicdomain/zero/1.0/) applies to the data made available in this article, unless otherwise stated in a credit line to the data. 
Keywords: Breast cancer, Capecitabine, Chemotherapy, Eribulin, Neutrophil-to-lymphocyte ratio, NLR, Prognostic factor

\section{Background}

Breast cancer is the most common malignancy among women worldwide [1], and patients with metastatic or recurrent human epidermal growth factor 2 (HER2)negative breast cancer have a poor prognosis, especially if they have previously received anthracycline or taxane treatment. The EMBRACE trial revealed that eribulin provided an improvement in overall survival (OS), relative to the physician's choice of treatment, in patients with metastatic or recurrent breast cancer [2]. However, another phase III study (Study 301) revealed that eribulin was not superior to capecitabine in terms of OS or progression-free survival (PFS) in this setting [3]. Thus, eribulin monotherapy or capecitabine monotherapy has become the most common real-world cytotoxic chemotherapy for patients who were previously treated using anthracycline or taxane [4], although no standard chemotherapy has been established for these patients if they do not have $B R C A$ loss-of-function mutations. Additional treatment options for these patients include vinorelbine or gemcitabine monotherapy.

Effective prognostic factors are needed to guide the selection of appropriate treatment for breast cancer, and reported prognostic factors include tumour size, stage, histological grade, lymph node status, hormone receptor (HR) status, and age [5]. However, these factors are typically used to predict the prognosis of patients with resectable breast cancer and thus are often not useful for guiding the selection of cytotoxic chemotherapy for metastatic or recurrent breast cancer. Thymidine phosphorylase expression has been reported as a biomarker of sensitivity to capecitabine treatment [6] and a predictive marker of docetaxel-modulated capecitabine treatment for patients with metastatic breast cancer [7]. However, using thymidine phosphorylase for guiding the appropriate treatment is not a straightforward approach as the expression is measured by immunohistochemistry of paraffin-embedded cancer tissues. Serum microRNA profiling is reportedly a biomarker for the effectiveness of eribulin and the development of new distant metastases in cases of metastatic breast cancer [8], although this biomarker is difficult to measure in a clinical setting.

Inflammation is a critical factor in tumour development and progression [9]. Thus, various studies have evaluated whether the prognosis of patients with breast cancer and other malignancies can be predicted using systemic inflammatory markers, such as lactate dehydrogenase (LDH) [10], C-reactive protein (CRP) [11,
12], albumin [13], the platelet-to-lymphocyte ratio (PLR) [14], absolute lymphocyte count (ALC) $[15,16]$, and the lymphocyte-to-monocyte ratio (LMR) [17-19]. The neutrophil-to-lymphocyte ratio (NLR) in peripheral blood, which is a marker of systemic immunity and inflammation, is also reportedly able to predict the prognosis of patients with solid tumours $[12,20]$ and breast cancer [21]. NLR has also been reported as a prognostic factor for patients with metastatic breast cancer [22]. Furthermore, relative to other chemotherapies, eribulin may play a relatively greater role in the relationship between the NLR and prognosis, as a low baseline NLR was significantly associated with improved outcomes among patients who received eribulin for locally advanced or metastatic breast cancer [23]. The NLR can predict outcomes among patients who received eribulin for metastatic breast cancer [24], and the NLR may be a more general prognostic factor, rather than a specific predictor of eribulin efficacy [16]. As the NLR is easily, rapidly, and readily determined using peripheral blood samples, it might be useful for guiding treatment for patients in clinical practice if it is confirmed to have prognostic value.

We are not aware of any reports regarding whether the NLR can predict outcomes among patients who receive capecitabine for metastatic or recurrent breast cancer. In addition, eribulin monotherapy or capecitabine monotherapy is the next cytotoxic chemotherapy option for breast cancer patients who have previously received anthracycline or taxane treatment, although it is unclear how to select the most appropriate option in this setting. Therefore, the present study aimed to evaluate whether the prognostic value of the NLR varies according to the use of eribulin or capecitabine, which could help guide treatment selection among patients who are eligible to receive eribulin monotherapy or capecitabine monotherapy for metastatic or recurrent breast cancer.

\section{Methods \\ Study cohort}

We retrospectively reviewed the medical records of patients with metastatic or recurrent breast cancer who had previously received anthracycline or taxane treatment at the National Cancer Center Hospital between 2007 and 2015. Patients with any type of simultaneous metastatic cancer were excluded. Patients were considered eligible if they had been treated using eribulin or capecitabine monotherapy as a first-line, second-line, or third-line chemotherapy for metastatic or recurrent 
breast cancer. Eribulin or capecitabine treatment was continued until tumour progression or the appearance of severe adverse events. The retrospective study protocol was approved by the institutional review board of the National Cancer Center Hospital (NCCH 2014-092) and complied with the Declaration of Helsinki.

\section{Variables}

Immunohistochemical staining at the time of the pathological diagnosis was performed to determine each patient's oestrogen receptor (ER), progesterone receptor (PgR), and HER2 statuses. Histopathological grading and immunohistochemical staining results for ER, PgR, and HER2 were interpreted based on previously reported guidelines [25]. Performance status (PS) was evaluated according to the Eastern Cooperative Oncology Group (ECOG) criteria. Tumour responses were assessed by the investigators according to the Response Evaluation Criteria in Solid Tumors (version 1.1). The overall response rate (ORR) was defined as the proportion of patients who achieved a complete response (CR) or partial response (PR). The OS interval was calculated from the start of eribulin or capecitabine treatment until death because of any cause or censoring at the last date of confirmed survival. The PFS interval was calculated from the start of treatment until the first instance of disease progression, death because of any cause, or censoring at the last date of confirmed survival without disease progression.

\section{Blood sample analysis}

Blood sample data were eligible for analysis if performed within 7 days before the start of eribulin or capecitabine monotherapy. NLR, LMR, and PLR were defined as the absolute neutrophil count divided by the absolute lymphocyte count, the absolute lymphocyte count divided by the absolute monocyte count, and the absolute platelet count divided by the absolute lymphocyte count, respectively.

\section{Statistical analysis}

Univariate analyses were performed to determine whether the ORR, OS, and PFS were associated with receptor status, surgical history, treatment history, albumin concentration (cut-off: $4.1 \mathrm{~g} / \mathrm{dL}$ ), age (cut-off: 60 years), LDH concentration (cut-off: $222 \mathrm{U} / \mathrm{L}$ ), CRP concentration (cut-off: $0.15 \mathrm{mg} / \mathrm{dL}$ ), NLR (cut-off: 3), ALC (cut-off: $1,500 / \mu \mathrm{L}$ ), LMR (cut-off: 5 ), and PLR (cutoff: 250). These cut-off values were selected based on previous reports [10-19]. Multivariable Cox proportional hazard models were used to analyse OS and PFS in two scenarios: (A) when the NLR was a definitive prognostic factor and using age, HR status, HER2 status, and NLR or (B) when the inflammatory markers that predicted
PFS in the univariate analyses were also included (age, HR status, HER2 status, ALC, NLR, LMR, and PLR). The ORR was analysed using the chi-squared test, while the OS and PFS outcomes were analysed using the Wilcoxon signed-rank sum test. Survival curves were also created using the Kaplan-Meier method. All statistical analyses were performed using JMP software (version 14.3.0 for Windows; SAS Institute Japan Inc., Cary, NC, USA), and results were considered statistically significant at a twosided $p$-value of $<0.05$.

\section{Results}

\section{Patient characteristics}

Between 2007 and 2015, we identified 125 patients who received eribulin and/or capecitabine for metastatic or recurrent breast cancer and had previously received an anthracycline or a taxane. The first-line, second-line, or third-line treatments for metastatic or recurrent breast cancer involved only eribulin (46 patients), only capecitabine (34 patients), or both eribulin and capecitabine (45 patients). All patients were female, and the median ages were 56years (range: $30-76$ years) for patients who received eribulin monotherapy and 59years (range: 36-74years) for patients who received capecitabine monotherapy. The patient characteristics are shown in Table 1 and Additional file 1. Relative to patients who received eribulin, patients who received capecitabine had a significantly lower LDH concentration and a significantly higher LMR. Capecitabine was administered at a significantly earlier line than eribulin, and patients who received capecitabine monotherapy had a significantly better response than patients who received eribulin monotherapy. Hormone therapy was significantly more often administered before chemotherapy for patients who received capecitabine than patients who received eribulin. The median follow-up period was 19.1 months.

\section{PFS and OS after starting eribulin or capecitabine monotherapy}

Eribulin was administered to 91 patients, with or without capecitabine monotherapy, and these patients had a median PFS of 4.4 months and a median OS of 16.2 months (Fig. 1). Capecitabine was administered to 79 patients, with or without eribulin monotherapy, and these patients had a median PFS of 8.5 months and OS of 33.0 months (Fig. 2). The PFS and OS curves stratified according to NLR ( $<3$ vs. $\geq 3$ ) revealed that a lower NLR was associated with significantly better outcomes in the eribulin and capecitabine groups (Figs. 3 and 4).

\section{Univariate analyses of ORR}

The univariate analyses revealed that, among patients who received eribulin monotherapy, a significantly 
Table 1 Patient characteristics

\begin{tabular}{|c|c|c|c|c|}
\hline & & $\begin{array}{l}\text { Eribulin } \\
(n=91)\end{array}$ & $\begin{array}{l}\text { Capecitabine } \\
(n=79)\end{array}$ & $p$ \\
\hline \multirow[t]{3}{*}{ Age in years, $\mathrm{n}(\%)$} & Median (range) & $56(30-76)$ & $59(36-74)$ & - \\
\hline & $\geq 60$ & $34(37)$ & $35(44)$ & 0.43 \\
\hline & $<60$ & $57(63)$ & $44(56)$ & \\
\hline \multirow[t]{2}{*}{ Sex, n (\%) } & Male & $0(0)$ & $0(0)$ & - \\
\hline & Female & $91(100)$ & $79(100)$ & \\
\hline \multirow[t]{3}{*}{ ECOG-PS, n (\%) } & 0 & $55(60)$ & $43(54)$ & 0.44 \\
\hline & 1 & $36(40)$ & $35(44)$ & \\
\hline & 2 & $0(0)$ & $1(1)$ & \\
\hline \multirow[t]{2}{*}{$H R, n(\%)$} & Positive & $71(78)$ & $70(89)$ & 0.1 \\
\hline & Negative & $20(22)$ & $9(11)$ & \\
\hline \multirow[t]{2}{*}{$E R, n(\%)$} & Positive & $68(75)$ & $65(82)$ & 0.27 \\
\hline & Negative & $23(25)$ & $14(18)$ & \\
\hline \multirow[t]{3}{*}{$\mathrm{PgR}, \mathrm{n}(\%)$} & Positive & $55(60)$ & $52(66)$ & 0.42 \\
\hline & Negative & $36(40)$ & $25(32)$ & \\
\hline & NA & $0(0)$ & $2(3)$ & - \\
\hline \multirow[t]{3}{*}{ HER2, n (\%) } & Positive & $5(6)$ & $2(3)$ & 0.45 \\
\hline & Negative & $85(93)$ & $75(95)$ & \\
\hline & NA & $1(1)$ & $2(3)$ & - \\
\hline Triple-negative, n (\%) & & $17(19)$ & $9(11)$ & 0.21 \\
\hline \multirow[t]{2}{*}{ Surgical history, n (\%) } & Positive & $83(91)$ & $72(91)$ & 1.0 \\
\hline & Negative & $8(9)$ & $7(9)$ & \\
\hline \multirow{2}{*}{$\begin{array}{l}\text { Neoadjuvant/adjuvant } \\
\text { chemotherapy, n (\%) }\end{array}$} & Positive & $76(84)$ & $63(80)$ & 0.56 \\
\hline & Negative & $15(16)$ & $16(20)$ & \\
\hline \multirow[t]{2}{*}{ Previous hormone therapy, $\mathrm{n}(\%)$} & Positive & $63(69)$ & $72(91)$ & 0.001 \\
\hline & Negative & $28(31)$ & $7(9)$ & \\
\hline \multirow{2}{*}{$\begin{array}{l}\text { Previous anthracycline, } \\
\text { n (\%) }\end{array}$} & Positive & $85(93)$ & $67(85)$ & 0.08 \\
\hline & Negative & $6(7)$ & $12(15)$ & \\
\hline \multirow[t]{2}{*}{ Previous taxane, n (\%) } & Positive & $88(97)$ & $73(92)$ & 0.31 \\
\hline & Negative & $3(3)$ & $6(8)$ & \\
\hline \multirow[t]{3}{*}{ Previous chemotherapy regimens, n (\%) } & 0 & $11(12)$ & $18(23)$ & 0.001 \\
\hline & 1 & $29(32)$ & $39(49)$ & \\
\hline & 2 & $51(56)$ & $22(28)$ & \\
\hline \multirow[t]{3}{*}{ Response, $n(\%)$} & $P R$ & $14(15)$ & $16(20)$ & 0.018 \\
\hline & SD & $43(47)$ & $49(62)$ & \\
\hline & $\mathrm{PD}$ & $34(37)$ & $14(18)$ & \\
\hline \multirow[t]{2}{*}{ Albumin, n (\%) } & $\geq 4.1 \mathrm{~g} / \mathrm{dL}$ & $55(60)$ & $51(65)$ & 0.64 \\
\hline & $<4.1 \mathrm{~g} / \mathrm{dL}$ & $36(40)$ & $28(35)$ & \\
\hline \multirow[t]{2}{*}{ LDH, $n(\%)$} & $<222 \mathrm{U} / \mathrm{L}$ & $38(42)$ & $48(61)$ & 0.01 \\
\hline & $\geq 222 \mathrm{U} / \mathrm{L}$ & $53(58)$ & $31(39)$ & \\
\hline \multirow[t]{2}{*}{ CRP, n (\%) } & $<0.15 \mathrm{mg} / \mathrm{dL}$ & $39(43)$ & $46(58)$ & 0.06 \\
\hline & $\geq 0.15 \mathrm{mg} / \mathrm{dL}$ & $52(57)$ & $33(42)$ & \\
\hline \multirow[t]{2}{*}{ NLR, n (\%) } & $<3$ & $65(71)$ & $56(71)$ & 1.0 \\
\hline & $\geq 3$ & $26(29)$ & $23(29)$ & \\
\hline \multirow[t]{2}{*}{ ALC, n (\%) } & $\geq 1,500 / \mu \mathrm{L}$ & $31(34)$ & $36(46)$ & 0.16 \\
\hline & $<1,500 / \mu \mathrm{L}$ & $60(66)$ & $43(54)$ & \\
\hline \multirow[t]{2}{*}{ LMR, n (\%) } & $\geq 5$ & $36(40)$ & $45(57)$ & 0.03 \\
\hline & $<5$ & $55(60)$ & $34(43)$ & \\
\hline \multirow[t]{2}{*}{ PLR, n (\%) } & $<250$ & $73(80)$ & $68(86)$ & 0.41 \\
\hline & $\geq 250$ & $18(20)$ & $11(14)$ & \\
\hline
\end{tabular}

ALC absolute lymphocyte count; CRP C-reactive protein; ECOG-PS Eastern Cooperative Oncology Group-performance status; $E R$ oestrogen receptor; HER2 human epidermal growth factor receptor 2; HR hormone receptor; $L D H$ lactate dehydrogenase; $L M R$ lymphocyte-to-monocyte ratio; NA not available; NLR neutrophil-tolymphocyte ratio; $P D$ progressive disease; $P g R$ progesterone receptor; $P L R$ platelet-to-lymphocyte ratio; $P R$ partial response; $S D$ stable disease 


\section{(A) PFS}

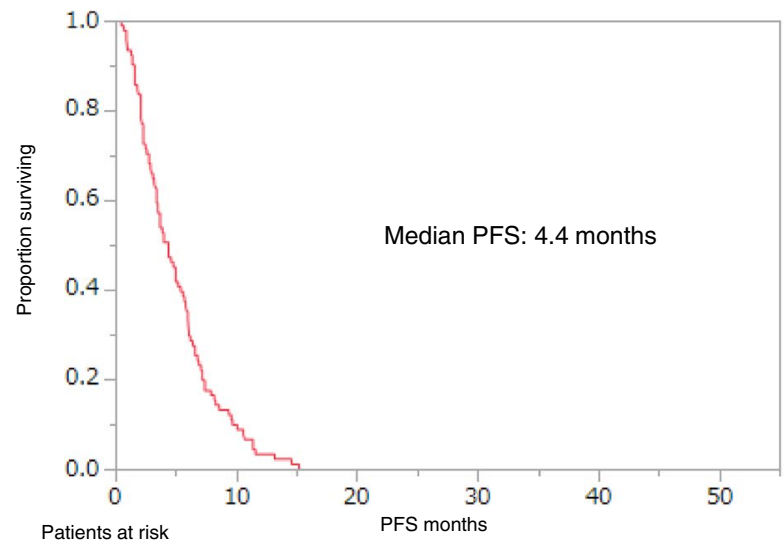

\section{(B) OS}

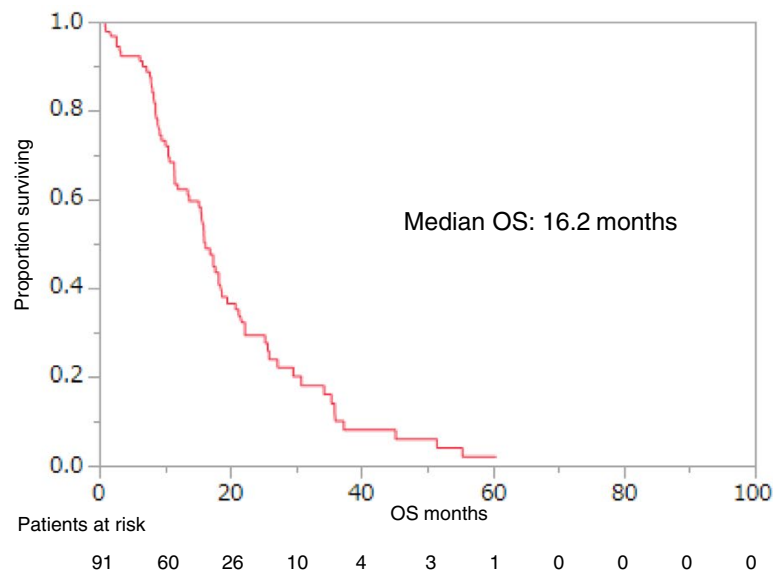

Fig. 1 Progression-free survival (PFS, A) and overall survival $(\mathrm{OS}, \mathbf{B})$ starting from first day of eribulin monotherapy

\section{(A) PFS}

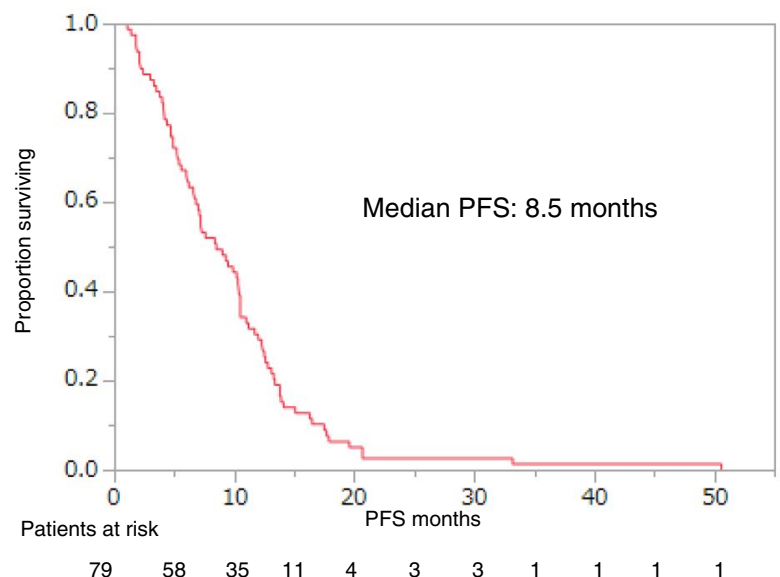

\section{(B) OS}

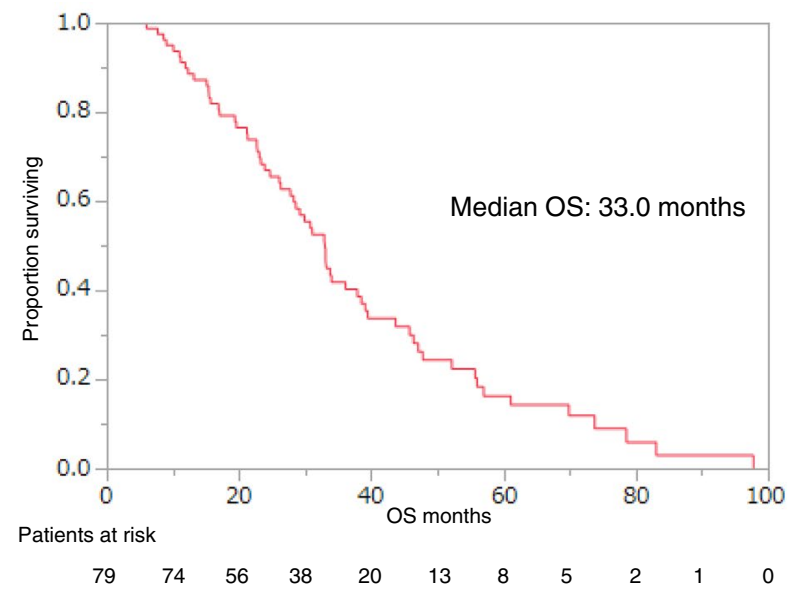

Fig. 2 Progression-free survival (PFS, A) and overall survival (OS, B) starting from first day of capecitabine monotherapy

better ORR was associated with $\mathrm{HR}+$ status $(p=0.034)$ and ER+ status $(p=0.018)$. Furthermore, among patients who received capecitabine monotherapy, significantly better ORR was associated with an NLR of $<3$ $(p=0.03)$ (Additional file 2).

\section{Univariate analyses of PFS}

The univariate analyses revealed that, among patients who received eribulin monotherapy, significantly better PFS was associated with an NLR $<3(p=0.011)$, a PLR of $<250(p=0.001)$, a surgical history $(p=0.006)$, and neoadjuvant/adjuvant chemotherapy $(p=0.045)$. Among patients who received capecitabine monotherapy, significantly better PFS was associated with an NLR of $<3(p=0.011), \mathrm{ER}+$ status $(p=0.029)$, a surgical history $(p=0.036)$, an ALC of $\geq 1,500 / \mu \mathrm{L}(p=0.013)$, an LMR of $\geq 5(p=0.001)$, and a PLR of $<250 \quad(p=0.037)$ (Additional file 3).

\section{Multivariable analyses of PFS}

When the NLR was treated as a definitive prognostic factor, an NLR of $<3$ predicted significantly better PFS 


\section{(A) Eribulin monotherapy}

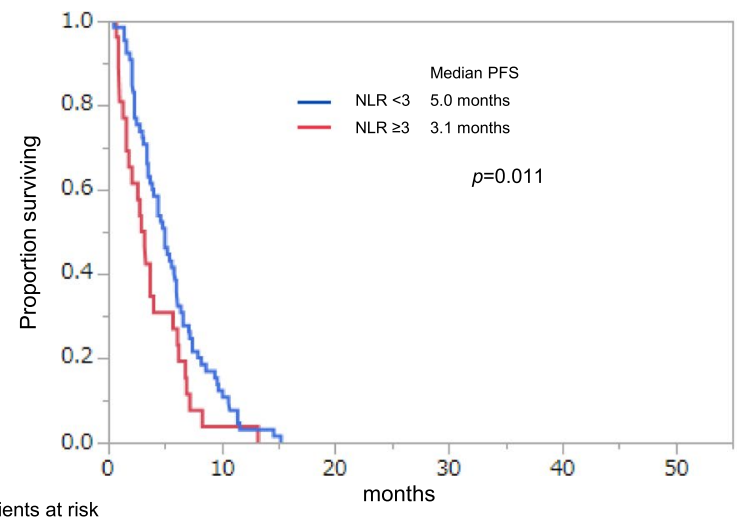

\section{(B) Capecitabine monotherapy}

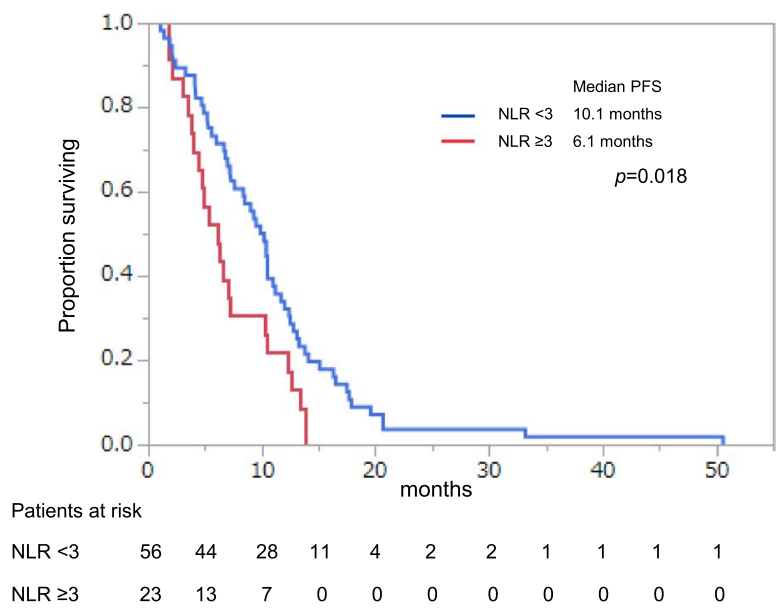

Fig. 3 Progression-free survival (PFS) stratified according to the neutrophil-to-lymphocyte ratio (NLR, $(<3$ vs. $\geq 3)$ starting from first day of eribulin monotherapy (A) or capecitabine monotherapy (B)

(A) Eribulin monotherapy

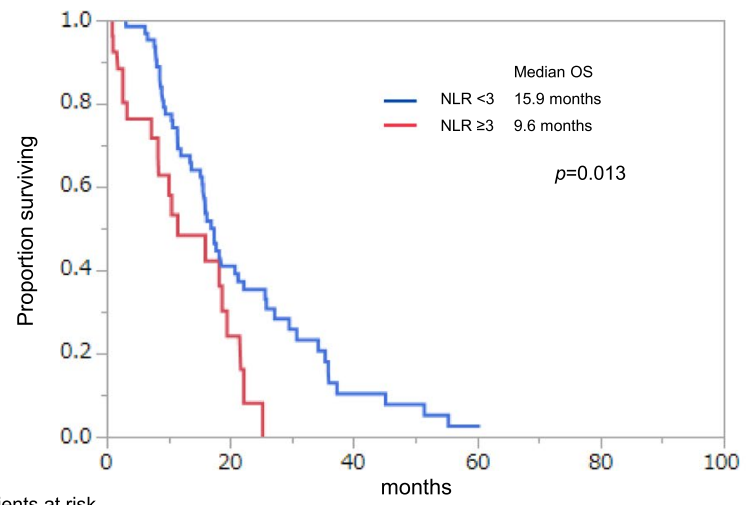

Patients at risk

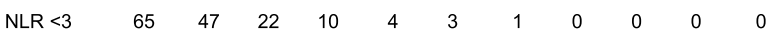

$\begin{array}{llllllllllll}\mathrm{NLR} \geq 3 & 26 & 13 & 4 & 0 & 0 & 0 & 0 & 0 & 0 & 0 & 0\end{array}$
(B) Capecitabine monotherapy

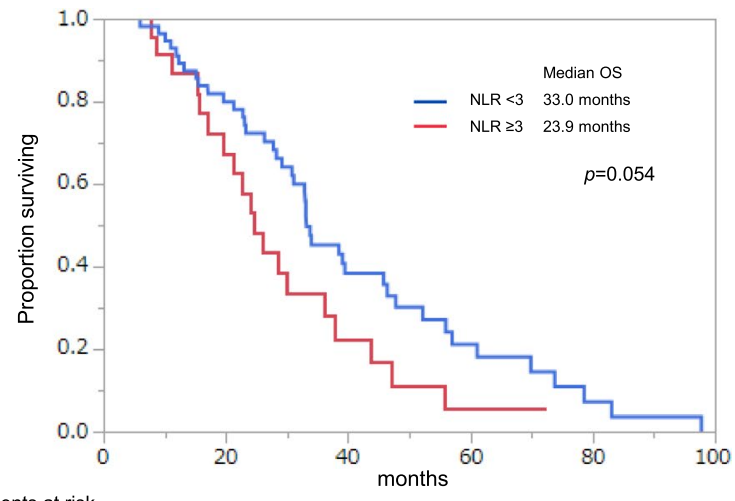

Patients at risk

$\begin{array}{llllllllllll}\text { NLR }<3 & 56 & 54 & 42 & 31 & 16 & 10 & 7 & 4 & 2 & 1 & 0\end{array}$

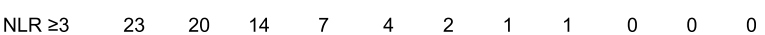

Fig. 4 Overall survival (OS) stratified according to the neutrophil-to-lymphocyte ratio (NLR, $(<3$ vs. $\geq 3)$ starting from first day of eribulin

monotherapy $(\mathbf{A})$ or capecitabine monotherapy $(\mathbf{B})$

among patients who received capecitabine monotherapy $(p=0.01)$ but not among patients who received eribulin monotherapy (Table 2). When inflammatory markers that predicted PFS were also included in the multivariable model, better PFS was predicted by an LMR of $\geq 5$ among patients who received capecitabine monotherapy $(p=0.03)$ and a PLR of $<250$ among patients who received eribulin monotherapy $(p=0.005)$ (Table 2$)$.

\section{Univariable analyses of OS}

The univariate analyses revealed that, among patients who received eribulin monotherapy, significantly better OS was associated with $\mathrm{HR}+$ status $(p=0.013), \mathrm{PgR}+$ status $(p=0.04)$, a surgical history $(p=0.024)$, previous hormone therapy $(p=0.01)$, an LDH concentration of $<222$ $\mathrm{U} / \mathrm{L}(p=0.001)$, an NLR of $<3(p=0.013)$, an LMR of $\geq 5$ $(p=0.013)$, and a PLR of $<250(p=0.002)$. Among patients who received capecitabine monotherapy, significantly 
Table 2 Multivariable analyses of progression-free survival

\begin{tabular}{|c|c|c|c|c|}
\hline & \multicolumn{2}{|l|}{ Eribulin $(n=91)$} & \multicolumn{2}{|c|}{ Capecitabine $(n=79)$} \\
\hline & $\begin{array}{l}\text { Hazard ratio } \\
(95 \% \mathrm{Cl})\end{array}$ & $p$ & $\begin{array}{l}\text { Hazard ratio } \\
(95 \% \mathrm{Cl})\end{array}$ & $p$ \\
\hline \multicolumn{5}{|c|}{ When the NLR was a definitive prognostic factor } \\
\hline Age ( $\geq 60$ years vs. $<60$ years) & $0.90(0.57-1.38)$ & 0.62 & $0.92(0.56-1.48)$ & 0.72 \\
\hline HR (positive vs. negative) & $0.91(0.54-1.62)$ & 0.74 & $1.22(0.62-2.71)$ & 0.58 \\
\hline HER2 (negative vs. positive) & $0.72(0.30-2.15)$ & 0.52 & $0.90(0.27-5.60)$ & 0.89 \\
\hline $\operatorname{NLR}(<3$ vs. $\geq 3)$ & $0.62(0.39-1.01)$ & 0.05 & $0.48(0.29-0.84)$ & 0.01 \\
\hline \multicolumn{5}{|c|}{ When inflammatory markers that predicted PFS were also included } \\
\hline Age ( $\geq 60$ years vs. $<60$ years) & $0.82(0.51-1.31)$ & 0.42 & $0.80(0.48-1.31)$ & 0.38 \\
\hline HR (positive vs. negative) & $0.94(0.55-1.70)$ & 0.83 & $1.26(0.59-2.97)$ & 0.57 \\
\hline HER2 (negative vs. positive) & $0.93(0.37-2.88)$ & 0.89 & $1.64(0.44-10.7)$ & 0.49 \\
\hline $\operatorname{ALC}(\geq 1,500 / \mu \mathrm{L}$ vs. $<1,500 / \mu \mathrm{L})$ & $0.96(0.57-1.61)$ & 0.89 & $0.68(0.38-1.22)$ & 0.19 \\
\hline $\operatorname{NLR}(<3$ vs. $\geq 3)$ & $0.69(0.41-1.20)$ & 0.18 & $0.86(0.40-1.91)$ & 0.70 \\
\hline LMR ( $\geq 5$ vs. $<5)$ & $1.20(0.73-1.97)$ & 0.47 & $0.55(0.33-0.94)$ & 0.03 \\
\hline PLR (<250 vs. $\geq 250)$ & $0.40(0.22-0.75)$ & 0.005 & $0.79(0.32-1.99)$ & 0.62 \\
\hline
\end{tabular}

$A L C$ absolute lymphocyte count; $C l$ confidence interval; $C R P$ C-reactive protein; $E R$ oestrogen receptor; HER2 human epidermal growth factor receptor 2; HR hormone receptor; $L D H$ lactate dehydrogenase; $L M R$ lymphocyte-to-monocyte ratio; $N L R$ neutrophil-to-lymphocyte ratio; $P F S$ progression-free survival; $P g R$ progesterone receptor; $P L R$ platelet-to-lymphocyte ratio

better OS was associated with an LDH concentration of $<222 \mathrm{U} / \mathrm{L}(p=0.002)$, a CRP concentration of $<0.15 \mathrm{mg}$ / $\mathrm{dL}(p=0.019)$, an NLR of $<3(p=0.037)$, and an LMR of $\geq 5(p=0.014)$ (Table 3$)$.

\section{Multivariable analyses of OS}

When the NLR was treated as a definitive prognostic factor, an NLR of $<3$ predicted significantly better OS among patients who received eribulin monotherapy $(p=0.03)$ but not among patients who received capecitabine monotherapy (Table 4). When inflammatory markers that predicted PFS were also included in the multivariable model, better OS was predicted by an LMR of $\geq 5$ among patients who received capecitabine monotherapy $(p=0.03)$ (Table 4).

\section{Discussion}

This retrospective study evaluated whether the NLR could predict oncological outcomes after eribulin or capecitabine monotherapy for patients with metastatic or recurrent breast cancer who had previously received an anthracycline or a taxane. The multivariable analysis revealed that an NLR of $<3$ predicted significantly better OS among patients who received eribulin, which agrees with previously reported results [23, 24]. However, among patients who received capecitabine, an NLR of $<3$ independently predicted better PFS but not better OS. Therefore, we cannot conclude that the NLR is useful for predicting OS among patients who receive capecitabine for metastatic or recurrent breast cancer.
Table 3 Univariate analyses of overall survival

\begin{tabular}{lll}
\hline & Eribulin $(\mathbf{n}=\mathbf{9 1})$ & $\begin{array}{l}\text { Capecitabine } \\
(\mathbf{n}=\mathbf{7 9})\end{array}$ \\
& $\boldsymbol{p}$ & $\mathbf{p}$ \\
\hline HR+ & $\mathbf{0 . 0 1 3}$ & 0.39 \\
ER+ & 0.13 & 0.05 \\
PgR+ & $\mathbf{0 . 0 4}$ & 0.84 \\
HER2- & 0.84 & 0.89 \\
Surgical history & $\mathbf{0 . 0 2 4}$ & 0.85 \\
Neoadjuvant/adjuvant chemo- & 0.63 & 0.58 \\
therapy & & \\
Previous hormone therapy & $\mathbf{0 . 0 1}$ & 0.8 \\
Previous anthracycline & 0.35 & 0.09 \\
Previous taxane & 0.13 & 0.28 \\
Albumin $\geq 4.1$ g/dL & 0.06 & 0.12 \\
Age $\geq 60$ years & 0.12 & 0.63 \\
LDH $<222 \mathrm{U} / \mathrm{L}$ & $\mathbf{0 . 0 0 1}$ & $\mathbf{0 . 0 0 2}$ \\
CRP $<0.15 \mathrm{mg} / \mathrm{dL}$ & 0.09 & $\mathbf{0 . 0 1 9}$ \\
NLR $<3$ & $\mathbf{0 . 0 1 3}$ & 0.037 \\
ALC $\geq 1,500 / \mu L$ & 0.11 & 0.06 \\
LMR $\geq 5$ & $\mathbf{0 . 0 1 4}$ \\
PLR $<250$ & $\mathbf{0 . 0 1 3}$ & 0.13 \\
\hline ALC & $\mathbf{0 . 0 0 2}$ &
\end{tabular}

$A L C$ absolute lymphocyte count; CRP C-reactive protein; $E R$ oestrogen receptor; HER2 human epidermal growth factor receptor type 2; HR hormone receptor; $L D H$ lactate dehydrogenase; $L M R$ lymphocyte-to-monocyte ratio; $N L R$ neutrophil-to-lymphocyte ratio; $P g R$ progesterone receptor; $P L R$ platelet-tolymphocyte ratio 
Table 4 Multivariable analyses of overall survival

\begin{tabular}{|c|c|c|c|c|}
\hline & \multicolumn{2}{|l|}{ Eribulin $(n=91)$} & \multicolumn{2}{|c|}{ Capecitabine $(n=79)$} \\
\hline & $\begin{array}{l}\text { Hazard ratio } \\
(95 \% \mathrm{Cl})\end{array}$ & $p$ & $\begin{array}{l}\text { Hazard ratio } \\
(95 \% \mathrm{Cl})\end{array}$ & $p$ \\
\hline \multicolumn{5}{|c|}{ When the NLR was a definitive prognostic factor } \\
\hline Age ( $\geq 60$ years $v s .<60$ years) & $0.74(0.44-1.21)$ & 0.24 & $0.92(0.53-1.56)$ & 0.76 \\
\hline HR (positive vs. negative) & $0.57(0.32-1.06)$ & 0.08 & $1.05(0.52-2.42)$ & 0.89 \\
\hline HER2 (negative vs. positive) & $0.87(0.25-5.44)$ & 0.85 & $1.08(0.23-19.3)$ & 0.94 \\
\hline $\operatorname{NLR}(<3$ vs. $\geq 3)$ & $0.53(0.30-0.95)$ & 0.03 & $0.56(0.32-1.01)$ & 0.05 \\
\hline \multicolumn{5}{|c|}{ When inflammatory markers that predicted PFS were also included } \\
\hline Age ( $\geq 60$ years $v s .<60$ years) & $0.60(0.35-1.01)$ & 0.06 & $0.74(0.41-1.30)$ & 0.3 \\
\hline HR (positive vs. negative) & $0.54(0.30-1.01)$ & 0.06 & $1.60(0.73-3.91)$ & 0.25 \\
\hline HER2 (negative vs. positive) & $1.05(0.30-6.69)$ & 0.94 & $2.34(0.45-43.1)$ & 0.36 \\
\hline $\operatorname{ALC}(\geq 1,500 / \mu \mathrm{L}$ vs. $<1,500 / \mu \mathrm{L})$ & $0.72(0.37-1.40)$ & 0.33 & $0.57(0.28-1.18)$ & 0.13 \\
\hline $\operatorname{NLR}(<3$ vs. $\geq 3)$ & $0.80(0.42-1.56)$ & 0.51 & $1.65(0.61-4.63)$ & 0.32 \\
\hline $\operatorname{LMR}(\geq 5$ vs. $<5)$ & $0.64(0.35-1.18)$ & 0.15 & $0.50(0.27-0.94)$ & 0.03 \\
\hline PLR (<250 vs. $\geq 250)$ & $0.63(0.33-1.30)$ & 0.2 & $0.50(0.18-1.35)$ & 0.17 \\
\hline
\end{tabular}

$A L C$ absolute lymphocyte count; $C R P$ C-reactive protein; $E R$ oestrogen receptor; $H E R 2$ human epidermal growth factor receptor 2; $H R$ hormone receptor; $L D H$ lactate dehydrogenase; $L M R$ lymphocyte-to-monocyte ratio; NLR neutrophil-to-lymphocyte ratio; PFS progression-free survival; PgR progesterone receptor; $P L R$ platelet-tolymphocyte ratio

We also evaluated various other potential prognostic factors, which revealed that a PLR of $<250$ predicted better PFS among patients who received eribulin monotherapy, while an LMR of $\geq 5$ predicted better PFS and OS among patients who received capecitabine monotherapy.

Effective prognostic factors, including thymidine phosphorylase and serum microRNA, have been identified to guide the selection of appropriate treatment for metastatic breast cancer [6-8]. In addition, NLR has been predicted to be a prognostic factor in clinical practice. Despite the ability of the NLR to predict outcomes after other chemotherapies, we did not observe an independent association between the NLR and OS among patients who received capecitabine for metastatic or recurrent breast cancer. This lack of an association may be related to the patients' characteristics and the NLR itself. For example, capecitabine was administered at a significantly earlier line than eribulin, and $>70 \%$ of patients received capecitabine as first-line or second-line treatment. Thus, the relationship between NLR and OS after capecitabine treatment might have been weakened by the effects of subsequent treatment lines. In addition, the NLR might only be a useful prognostic factor for patients with ER-negative and HER2-negative breast cancer [21], while the present study included patients with various HR subtypes of breast cancer. Furthermore, the NLR might not accurately reflect the patient's inflammatory status in this setting, as chemotherapy-induced myelosuppression can lead to neutropenia. In contrast, platelets or monocytes are less sensitive to myelosuppression (vs. neutrophils). Thus, the PLR or LMR might be more accurate inflammatory markers and better able to predict survival outcomes in this setting. Moreover, overexpression of immune-related genes might not predict the response to capecitabine monotherapy [26], which could suggest that the NLR (as an inflammatory marker) might not be an appropriate prognostic factor for capecitabinetreated patients. Further studies are needed to identify more appropriate non-inflammatory markers that can be used to predict survival outcomes after starting capecitabine monotherapy in this setting.

The present study has three important limitations. First, the sample size was small, which could limit the power of the analyses. Second, many cases were missing information regarding the histological grade (which is generally considered a prognostic factor), and we were unable to consider this variable in the analyses. Finally, we were unable to evaluate prognostic factors such as thymidine phosphorylase and serum microRNA.

\section{Conclusions}

To the best of our knowledge, this is the first study to evaluate whether the NLR can predict outcomes after first-line, second-line, or third-line capecitabine monotherapy among patients with metastatic or recurrent breast cancer. The results revealed that an NLR of $<3$ independently predicted better PFS but not better OS. Therefore, we cannot conclude that the NLR is useful for predicting OS among patients who receive capecitabine for metastatic or recurrent breast cancer. Additional research is needed to identify prognostic factors 
that can guide the selection of eribulin or capecitabine treatment in this setting.

\begin{abstract}
Abbreviations
ALC: absolute lymphocyte count; CR: complete response; CRP: C-reactive protein; ECOG-PS: Eastern Cooperative Oncology Group-performance status; ER: oestrogen receptor; HER2: human epidermal growth factor receptor 2; HR: hormone receptor; LDH: lactate dehydrogenase; LMR: lymphocyte-to-monocyte ratio; NA: not available; NLR: neutrophil-to-lymphocyte ratio; ORR: overall response rate; OS: overall survival; PD: progressive disease; PFS: progressionfree survival; PgR: progesterone receptor; PLR: platelet-to-lymphocyte ratio; PR: partial response; SD: stable disease.
\end{abstract}

\section{Supplementary Information}

The online version contains supplementary material available at https://doi. org/10.1186/s12885-021-09112-9.

Additional file 1. Patient characteristics excluding duplicate cases.

Additional file 2. Univariate analyses of overall response rate.

Additional file 3. Univariate analyses of progression-free survival.

\section{Acknowledgements}

The authors thank the patients and participating investigators.

\section{Authors' contributions}

All authors listed in the manuscript have sufficiently contributed to the project to qualify as authors, and all qualified authors are listed in the author byline. ST and TS designed the study. The first draft of the manuscript was written by ST and TS. NT contributed to the data curation. ST, TS, NT, SY, TO, YK, HO, TN, MT, $\mathrm{KS}, \mathrm{EN}$, and $\mathrm{KY}$ drafted the manuscript and approved the final version of the manuscript.

\section{Funding}

None.

\section{Availability of data and materials}

To protect patient privacy, detailed patient records are not available. All relevant data are included in the manuscript and its associated files.

\section{Declarations}

\section{Ethics approval and consent to participate}

The retrospective study protocol was approved by the National Cancer Center Research Ethics Review Committee (Tokyo, Japan; NCCH 2014-092), which waived the requirement for informed consent. Patients are able to optout of the research use of their data at the hospital's website. All research was conducted in accordance with the Declaration of Helsinki.

\section{Consent for publication}

Not applicable.

\section{Competing interests}

The authors declare that they have no competing interests.

Received: 19 March 2021 Accepted: 14 December 2021

Published online: 14 January 2022

\section{References}

1. Bray F, Ferlay J, Soerjomataram I, Siegel RL, Torre LA, Jemal A. Global cancer statistics 2018: GLOBOCAN estimates of incidence and mortality worldwide for 36 cancers in 185 countries. CA Cancer J Clin. 2018;68:394-424.
2. Cortes J, O'Shaughnessy J, Loesch D, Blum JL, Vahdat LT, Petrakova K, et al. Eribulin monotherapy versus treatment of physician's choice in patients with metastatic breast cancer (EMBRACE): A phase 3 open-label randomised study. Lancet. 2011;377:914-23.

3. Kaufman PA, Awada A, Twelves C, Yelle L, Perez EA, Velikova G, et al. Phase III open-label randomized study of eribulin mesylate versus capecitabine in patients with locally advanced or metastatic breast cancer previously treated with an anthracycline and a taxane. J Clin Oncol. 2015;33:594-601.

4. lizumi S, Shimoi T, Tsushita N, Bun S, Shimomura A, Noguchi E, et al. Efficacy and safety of eribulin in patients with locally advanced or metastatic breast cancer not meeting trial eligibility criteria: A retrospective study. BMC Cancer. 2017;17:819.

5. From the American Association of Neurological Surgeons (AANS), American Society of Neuroradiology (ASNR), Cardiovascular and Interventional Radiology Society of Europe (CIRSE), Canadian Interventional Radiology Association (CIRA), Congress of Neurological Surgeons (CNS), European Society of Minimally Invasive Neurological Therapy (ESMINT), European Society of Neuroradiology (ESNR), European Stroke Organization (ESO), Society for Cardiovascular Angiography and Interventions (SCAI), Society of Interventional Radiology (SIR), Society of Neurolnterventional Surgery (SNIS), and World Stroke Organization (WSO), Sacks D, Baxter B, Campbell BCV, Carpenter JS, Cognard C, et al. From the American Association of Neurological Surgeons (AANS) Multisociety Consensus Quality Improvement Revised Consensus Statement for Endovascular Therapy of Acute Ischemic Stroke. Int J Stroke. 2018;13:612-32.

6. Andreetta C, Puppin C, Minisini A, Valent F, Pegolo E, Damante G, et al. Thymidine phosphorylase expression and benefit from capecitabine in patients with advanced breast cancer. Ann Oncol. 2009;20:265-71.

7. Puglisi F, Cardellino GG, Crivellari D, Di Loreto C, Magri MD, Minisini AM, et al. Thymidine phosphorylase expression is associated with time to progression in patients receiving low-dose, docetaxel-modulated capecitabine for metastatic breast cancer. Ann Oncol. 2008;19:1541-6.

8. Satomi-Tsushita N, Shimomura A, Matsuzaki J, Yamamoto Y, Kawauchi J, Takizawa S, et al. Serum microRNA-based prediction of responsiveness to eribulin in metastatic breast cancer. PLOS ONE. 2019;14:e0222024.

9. Grivennikov SI, Greten FR, Karin M. Immunity, inflammation, and cancer. Cell. 2010;140:883-99.

10. Pelizzari G, Basile D, Zago S, Lisanti C, Bartoletti M, Bortot L, et al. Lactate dehydrogenase $(\mathrm{LDH})$ response to first-line treatment predicts survival in metastatic breast cancer: First clues for A cost-effective and dynamic biomarker. Cancers (Basel). 2019;11(9).

11. Shimura T, Shibata M, Gonda K, Murakami Y, Noda M, Tachibana K, et al. Prognostic impact of interleukin-6 and C-reactive protein on patients with breast cancer. Oncol Lett. 2019;17:5139-46.

12. Artaç M, Uysal M, Karaağaç M, Korkmaz L, Er Z, Güler T, et al. Prognostic impact of neutrophil/lymphocyte ratio, platelet count, CRP, and albumin levels in metastatic colorectal cancer patients treated with FOLFIRI-bevacizumab. J Gastrointest Cancer. 2017;48:176-80.

13. Fujii T, Tokuda S, Nakazawa Y, Kurozumi S, Obayashi S, Yajima R, et al. Implications of low serum albumin as a prognostic factor of long-term outcomes in patients With breast cancer. In Vivo. 2020;34:2033-6.

14. Hirahara T, Arigami T, Yanagita S, Matsushita D, Uchikado Y, Kita Y, et al. Combined neutrophil-lymphocyte ratio and platelet-lymphocyte ratio predicts chemotherapy response and prognosis in patients with advanced gastric cancer. BMC Cancer. 2019;19:672.

15. Araki K, Ito Y, Fukada I, Kobayashi K, Miyagawa Y, Imamura M, et al. Predictive impact of absolute lymphocyte counts for progression-free survival in human epidermal growth factor receptor 2-positive advanced breast cancer treated with pertuzumab and trastuzumab plus eribulin or nabpaclitaxel. BMC Cancer. 2018;18:982.

16. Miyoshi Y, Yoshimura Y, Saito K, Muramoto K, Sugawara M, Alexis K, et al. High absolute lymphocyte counts are associated with longer overall survival in patients with metastatic breast cancer treated with eribulin-but not with treatment of physician's choice-in the EMBRACE study. Breast Cancer. 2020;27:706-15.

17. Hu RJ, Liu Q, Ma JY, Zhou J, Liu G. Preoperative lymphocyte-to-monocyte ratio predicts breast cancer outcome: A meta-analysis. Clin Chim Acta. 2018;484:1-6. 
18. He J, Lv P, Yang X, Chen Y, Liu C, Qiu X. Pretreatment lymphocyte to monocyte ratio as a predictor of prognosis in patients with early-stage triple-negative breast cancer. Tumour Biol. 2016;37:9037-43.

19. Ji H, Xuan Q, Yan C, Liu T, Nanding A, Zhang Q. The prognostic and predictive value of the lymphocyte to monocyte ratio in luminal-type breast cancer patients treated with CEF chemotherapy. Oncotarget. 2016;7:34881-9.

20. Lopes M, Carvalho B, Vaz R, Linhares P. Influence of neutrophil-lymphocyte ratio in prognosis of glioblastoma multiforme. J Neurooncol. 2018;136:173-80.

21. Ethier JL, Desautels D, Templeton A, Shah PS, Amir E. Prognostic role of neutrophil-to-lymphocyte ratio in breast cancer: A systematic review and meta-analysis. Breast Cancer Res. 2017;19:2.

22. Gerratana L, Basile D, Toffoletto B, Bulfoni M, Zago S, Magini A, et al. Biologically driven cut-off definition of lymphocyte ratios in metastatic breast cancer and association with exosomal subpopulations and prognosis. Sci Rep. 2020;10:7010.

23. Miyagawa Y, Araki K, Bun A, Ozawa H, Fujimoto Y, Higuchi T, et al. Significant association Between low baseline neutrophil-to-lymphocyte ratio and improved progression-free survival of patients With locally advanced or metastatic breast cancer treated With eribulin but not With nabpaclitaxel. Clin Breast Cancer. 2018;18:400-9.

24. Ueno A, Maeda R, Kin T, Ito M, Kawasaki K, Ohtani S. Utility of the absolute lymphocyte count and neutrophi//ymphocyte ratio for predicting survival in patients with metastatic breast cancer on eribulin: A real-world observational study. Chemotherapy. 2019;64:259-69.

25. Shimoi T, Yoshida M, Kitamura Y, Yoshino T, Kawachi A, Shimomura A, et al. Tert promoter hotspot mutations in breast cancer. Breast Cancer. 2018;25:292-6.

26. Foukakis T, Lövrot J, Matikas A, Zerdes I, Lorent J, Tobin N, et al. Immune gene expression and response to chemotherapy in advanced breast cancer. Br J Cancer. 2018;118:480-8.

\section{Publisher's Note}

Springer Nature remains neutral with regard to jurisdictional claims in published maps and institutional affiliations.

Ready to submit your research? Choose BMC and benefit from:

- fast, convenient online submission

- thorough peer review by experienced researchers in your field

- rapid publication on acceptance

- support for research data, including large and complex data types

- gold Open Access which fosters wider collaboration and increased citations

- maximum visibility for your research: over 100M website views per year

At BMC, research is always in progress.

Learn more biomedcentral.com/submissions 